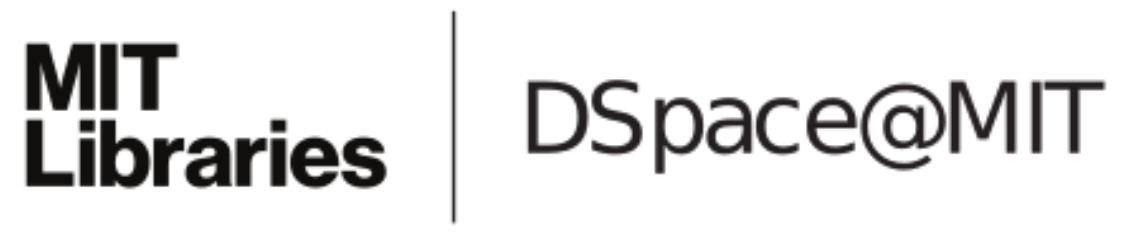

\author{
MIT Open Access Articles
}

A Ge-on-Si laser for electronic-photonic integration

The MIT Faculty has made this article openly available. Please share how this access benefits you. Your story matters.

Citation: Sun, Xiaochen, et al. "A Ge-on-Si laser for electronic-photonic integration." Lasers and Electro-Optics, 2009 and 2009 Conference on Quantum electronics and Laser Science Conference. CLEO/QELS 2009. Conference on. 2009. 1-2. ๑2009 IEEE.

Publisher: Institute of Electrical and Electronics Engineers

Persistent URL: http://hdl.handle.net/1721.1/61670

Version: Final published version: final published article, as it appeared in a journal, conference proceedings, or other formally published context

Terms of Use: Article is made available in accordance with the publisher's policy and may be subject to US copyright law. Please refer to the publisher's site for terms of use. 


\title{
CTuY1.pdf
}

\section{A Ge-on-Si Laser for Electronic-Photonic Integration}

\author{
Xiaochen Sun, Jifeng, Liu, Lionel C. Kimerling, and Jurgen Michel \\ Microphotonic Center, Massachusetts Institute of Technology, Cambridge, MA 02139
}

\begin{abstract}
We demonstrate room temperature photoluminescence and optical gain from the direct band gap transition of tensile strained n-type Ge-on-Si around 1600 $\mathrm{nm}$, which can be applied to a Si-based laser for optical interconnects and communications.
\end{abstract}

(C)2009 Optical Society of America

OCIS codes: (250.0250) Optoelectronics; (250.5960) Semiconductor lasers

A Si-based laser capable of monolithic integration is the last missing link in the chain to realize electronic-photonic integration which is a potential solution to the bandwidth limitation and power consumption in traditional electronic integrated circuits (ICs). Ge has been proposed as a very promising candidate for such a Si-based laser [1]. Ge has been widely applied to high mobility complementary metal oxide semiconductor (CMOS) transistors in Si ICs for years. Capable of monolithic integration on the Si platform, Ge-based devices require far less fabrication cost than hybrid III-V lasers on Si [2,3]. Moreover, the Ge direct gap emission is around $1550 \mathrm{~nm}$ in wavelength, coincident with the wavelength band used for optical interconnects and fiber based optical communications. However, the Ge direct gap emission is limited by the lack of excited electrons in the direct valley of the conduction band under injection since most electrons occupy the indirect valleys with lower energy states according to the Fermi distribution. This situation can be improved by introducing in-plane biaxial tensile stress in Ge films to reduce the energy difference between the direct and the indirect band gaps. In addition, doping Ge with n-type impurities fills the indirect valleys with extrinsic electrons and raises the Fermi level, which further facilitates the occupation of excited electrons in the direct valley under injection. Theoretical calculations have shown that a combination of $0.25 \%$ tensile strain and mid $10^{19} \mathrm{~cm}^{-3} \mathrm{n}$-type doping is sufficient to get direct gap light emission from Ge that is as efficient as that of III-V materials [1]. In this paper, we report experimental results on the room temperature photoluminescence (PL) and optical gain from the direct gap transition of such tensile strained n-type Ge-on-Si.

Ge films with $0.25 \%$ tensile strain and various phosphorus doping concentrations are epitaxially grown on Si by using ultra-high vacuum chemical vapor deposition (UHVCVD). The Ge films are nearly fully relaxed at the growth temperature above $650 \mathrm{C}$, and tensile strain is accumulated upon cooling to room temperature due to the larger thermal expansion coefficient of Ge than that of $\mathrm{Si}[4,5]$. The direct band gap is red-shifted to $\sim 0.765 \mathrm{eV}(1620 \mathrm{~nm})$ due to the tensile strain. The phosphorus donors are incorporated during the epitaxial growth by introducing $\mathrm{PH}_{3}$ gas and the doping level is determined by the $\mathrm{GeH}_{4} / \mathrm{PH}_{3}$ ratio.

Room temperature PL at the direct gap energy is observed for all n-type doped Ge films. Ge films with doping level of $1 \times 10^{19} \mathrm{~cm}^{-3}$ exhibit an over 50 times enhancement in PL intensity compared to undoped films, as shown in Fig. 1(a). It can be seen in Fig. 1(b) that the integral PL intensity increases with the active doping concentration measured by Hall effect. This result agrees with theoretical calculations of the direct band gap PL enhancement due to the indirect valley filling effect. A room temperature pump-probe measurement has been performed to study the absorption/gain spectrum of the materials under steady state continuous-wave pumping. An optical bleaching effect, i.e. absorption reduction due to optical pumping, was observed above the direct band gap energy from this measurement, indicating the potential of population inversion. This optical bleaching effect also increases with increasing doping concentration in Ge at the same pumping level due to the indirect valley filling effect. To achieve better carrier confinement, a Ge mesa structure is formed by using a selective growth approach [6]. A scanning electron microscope (SEM) image of a Ge mesa structure is shown in Fig. 2(a). The facets on the edge of the mesa indicate the single-crystal nature of the Ge mesa. Pump-probe measurement on $500 \mu \mathrm{m}^{2}$ Ge mesas with $0.25 \%$ tensile strain and $10^{19} \mathrm{~cm}^{-3} \mathrm{n}$-type doping shows an optical gain (negative absorption) of up to $56 \pm 25 \mathrm{~cm}^{-1}(240 \pm 100 \mathrm{~dB} / \mathrm{cm})$ near the direct gap 


\section{CTuY1.pdf}

energy plotted in Fig 2(b). These results indicate that with tensile-strained $\mathrm{n}^{+} \mathrm{Ge}-\mathrm{on}-\mathrm{Si}$ as the gain medium and a carefully designed optical resonator for feedback, lasing from Si-based Ge could be achieved in the near future. Fabrication of a high Q resonator is in progress to demonstrate an optically pumped Ge laser on Si.

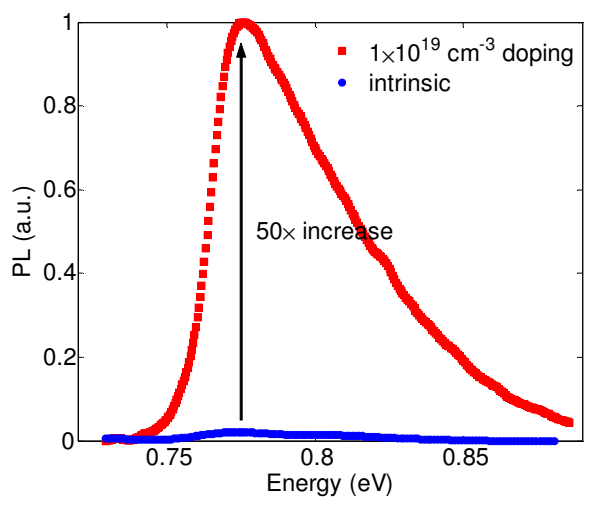

1(a)

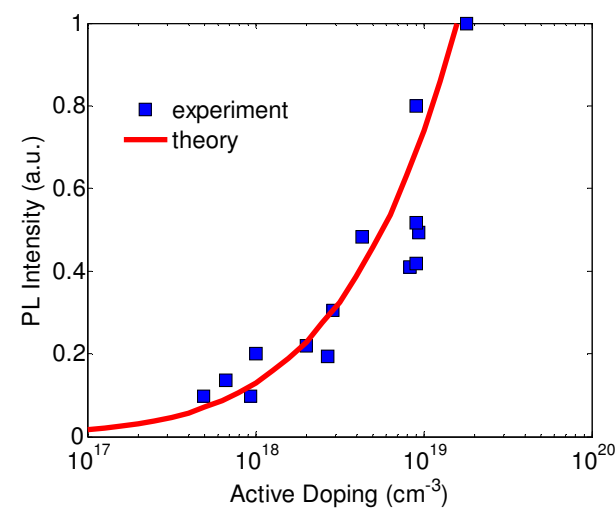

$1(\mathrm{~b})$

Fig. 1. (a) Comparison of room temperature direct gap PL spectra of $0.25 \%$ tensile strained Ge with $1 \times 10^{19} \mathrm{~cm}^{-3} \mathrm{n}$-type doping and without doping. (b) Integral PL intensity as a function of active n-type doping level and its comparison to theoretical calculations. The injection level is estimated to be $\Delta \mathrm{n}=\Delta \mathrm{p} \sim 10^{17} \mathrm{~cm}^{-3}$ for these measurements.

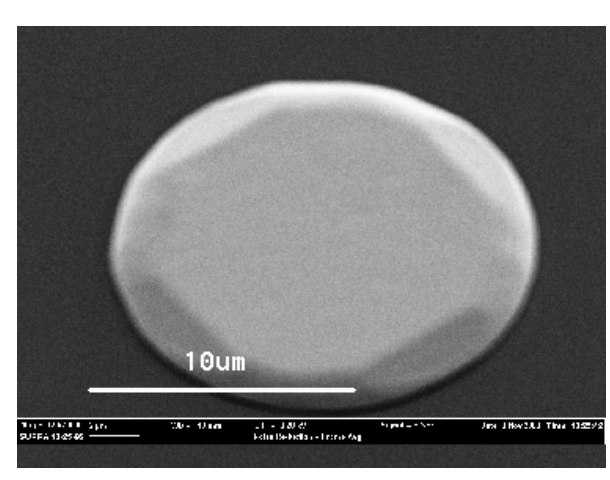

2(a)

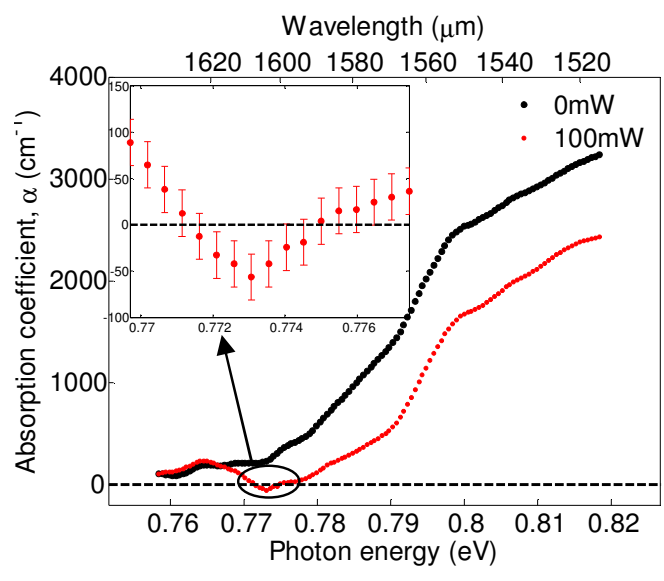

2(b)

Fig. 2. (a) SEM image of a Ge mesa structure with $15 \mu \mathrm{m}$ in diameter. The facets on the edges of the mesa are due to growth rate differences for several crystallographic orientations during the epitaxial trench growth. (b) Absorption spectra of the $\mathrm{n}+\mathrm{Ge}$ mesa sample under 0 and $100 \mathrm{~mW}$ optical pumping. Negative absorption coefficients corresponding to the onset of optical gain are observed in the wavelength range of 1600-1608 nm, as shown in the inset. The error bars given in the inset take into account the accuracy of both the transmittance measurement and the film thickness measurement.

\section{References}

[1] J. Liu, X. Sun, D. Pan, X. Wang, L. C. Kimerling, T. L. Koch, and J. Michel, Opt. Express, 15, 11272-11277 (2007)

[2] A. W. Fang, H. Park, O. Cohen, R. Jones, M. J. Paniccia, and J. E. Bowers, Opt. Express, 14, 9203-9210 (2006)

[3] M. E. Groenert, C. W. Leitz, A. J. Pitera, V. Yang, H. Lee, R. Ram and E. A. Fitzerald, J. Appl. Phys., 93, 362-367, (2003)

[4] Y. Ishkawa, K. Wada, D. D. Cannon, J. Liu, H. Luan and L. C. Kimerling, Appl. Phys. Lett., 82, 2044-2046, (2003)

[5] J. Liu, D. D. Cannon. Y. Ishikawa, K, Wada, D, T. Danielson, S, Jongthammanurak, J. Michel, and L. C. Kimerling, Phys. Rev. B., 70, 155309 (2004)

[6] J. Liu, J. Michel, W. Giziewicz, Dong Pan, K. Wada, D. D. Cannon, S. Jongthammanurak, D. T. Danielson, L. C. Kimerling, J. Chen, F. O. Ilday, F. X. Kartner, and J. Yasaitis, Appt. Phys. Lett., 87, 103510 (2005) 